\title{
A closure model with plumes
}

\section{The solar convection ${ }^{\star}$}

\author{
K. Belkacem ${ }^{1}$, R. Samadi ${ }^{1}$, M. J. Goupil ${ }^{1}$, and F. Kupka ${ }^{2}$ \\ 1 Observatoire de Paris, LESIA, CNRS UMR 8109, 92195 Meudon, France \\ e-mail: Kevin.Belkacem@obspm.fr \\ 2 Max-Planck-Institute for Astrophysics, Karl-Schwarzschild Str. 1, 85741 Garching, Germany
}

Received 5 April 2006 / Accepted 18 August 2006

ABSTRACT

\begin{abstract}
Context. Oscillations of stellar $p$ modes, excited by turbulent convection, are investigated. In the uppermost part of the solar convection zone, radiative cooling is responsible for the formation of turbulent plumes, hence the medium is modelled with downdrafts and updrafts.

Aims. We take into account the asymmetry of the up- and downflows created by turbulent plumes through an adapted closure model. In a companion paper, we apply it to the formalism of excitation of solar $p$ modes developed by Samadi \& Goupil (2001).

Methods. Using results from 3D numerical simulations of the uppermost part of the solar convection zone, we show that the two-scale mass-flux model (TFM) is valid only for quasi-laminar or highly skewed flows (Gryanik \& Hartmann 2002) and does not reproduce turbulent properties of the medium such as velocity-correlation products. We build a generalized two-scale mass-flux Model (GTFM) model that takes both the skew introduced by the presence of two flows and the effects of turbulence in each flow into account. In order to apply the GTFM to the solar case, we introduce the plume dynamics as modelled by Rieutord \& Zahn (1995) and construct a closure model with plumes (CMP).

Results. The CMP enables expressing the third- and fourth-order correlation products in terms of second-order ones. When compared with 3D simulation results, the CMP improves the agreement for the fourth-order moments by a factor of two approximately compared with the use of the quasi-normal approximation or a skewness computed with the classical TFM.

Conclusions. The asymmetry of turbulent convection in the solar case has an important impact on the vertical-velocity fourth-order moment, which has to be accounted for by models. The CMP is a significant improvement and is expected to improve the modelling of solar $p$-mode excitation.
\end{abstract}

Key words. convection - turbulence - Sun: oscillations

\section{Introduction}

In the uppermost part of the solar convective zone, turbulent entropy fluctuations and motions of eddies drive acoustic oscillations. 3D numerical simulations of the stellar turbulent outer layers have been used to compute the excitation rates of solarlike oscillation modes (Nordlund \& Stein 2001). As an alternative approach, semi-analytical modelling can provide an understanding of the physical processes involved in the excitation of $p$ modes: in this case, it is indeed rather easy to isolate the different physical mechanisms at work in the excitation process and to assess their effects. Various semi-analytical approaches have been developed by several authors (Goldreich \& Keeley 1977; Goldreich et al. 1994; Balmforth 1992; Samadi \& Goupil 2001); they differ from each other by the nature of the assumed excitation sources, by the assumed simplifications and approximations, and also by the way the turbulent convection is described (see the review by Stein et al. 2004). Among the different theoretical approaches, that of Samadi \& Goupil (2001) includes a detailed treatment of turbulent convection, which enables us to investigate different assumptions about turbulent convection in the outer layers of stars (Samadi et al. 2005). In this approach, the analytical expression for the acoustic power supplied to the

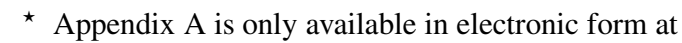
http: //www . aanda.org $p$ modes involves fourth-order correlation functions of the turbulent Reynolds stress and the entropy source term, which for the sake of simplicity are expressed in terms of second-order moments by means of a closure model.

The most commonly used closure model at the level of fourth-order moments (FOM) is the Quasi-Normal Approximation (QNA), which is valid for a Gaussian probability distribution function (see Lesieur 1997) and was first introduced by Millionshchikov (1941). The QNA is rather simple and convenient to implement. However, Ogura (1963) has shown that such a closure could lead to part of the kinetic energy spectrum becoming negative. In this paper, we confirm the results of Kupka \& Robinson (2006, hereafter KR2006), namely that this approximation indeed provides a poor description of the physical processes involved in solar turbulent convection.

Mass flux models (e.g., Randall et al. 1992; Abdella \& McFarlane 1997) explicitly take the effects of updrafts and downdrafts on the correlation products into account. The presence of two well-defined flow directions then introduces an additional contribution when averaging the fluctuating quantities, since averages of fluctuating quantities over each individual flow differ from averages over the total flow. For applications in atmospheric sciences, the mass-flux model for convection has recently been improved by Gryanik \& Hartmann (2002, hereafter GH2002). Their motivation has been to account for the fact 
that horizontal scales of temperature and velocity fluctuations are different (hence their improvements lead to a "two-scale mass-flux model" (TFM)) as well as to understand and measure the effects of the skewness of their distribution. According to GH2002, mass-flux models, which also include the TFM, underestimate the FOM by as much as $70 \%$. Therefore, such models clearly miss some important physical effects present in convective flows. Gryanik \& Hartmann (2002) and Gryanik et al. (2005) studied the asymptotic limits of TFM which led the authors to propose an interpolation between the QNA and the limit of large skewness provided by the TFM. This new parametrization permits a much better description of the FOM for convection in the atmosphere of the Earth (GH2002). We show that for their parametrization to be applicable to the case of solar convection, a more realistic estimate for the skewnesses of velocity and temperature fluctuations is required than that provided by the TFM itself (Sect. 2).

The parametrization of $\mathrm{GH} 2002$ requires the knowledge of the skewnesses and second-order moments to compute FOM. These have to be provided either by measurements, by another model, or by numerical simulations. In the present paper we do not aim to construct a complete model to compute these quantities, which is the goal of the Reynolds stress approach (e.g., Canuto 1992; Canuto \& Dubovikov 1998). Rather, we aim to analyze the shortcomings of the TFM and suggest improvements using numerical simulations of solar convection as a guideline. The conclusions drawn from this analysis are used to derive a model for fourth-order moments in terms of second-order moments that can be used in computations of solar $p$-mode excitation rates.

To proceed with the latter, we developed a formulation of the TFM that takes the effects of turbulence in each flow into account. This generalized TFM model (hereafter GTFM) is useful for both the superadiabatic and adiabatic outer solar layers. This formulation can actually be applied in other contexts than just the excitation of solar $p$ modes as long as the convective system is composed of two flows.

The GTFM is more general and realistic than the TFM, but it requires the knowledge of additional properties of both the turbulent upwards and downwards flows. We choose to determine these properties by means of a plume model. Turbulent plumes are created at the upper boundary of the convection zone, where radiative cooling becomes dominant and where the flow reaches the stable atmosphere. In this region the updrafts become cooler and stop their ascent. This cooler flow is more dense than its environment and it triggers the formation of turbulent plumes (Stein \& Nordlund 1998). As shown by Rieutord \& Zahn (1995), these structures drive the dynamics of the flow; hence, to construct a closure model, we study the plume dynamics developed by Rieutord \& Zahn (1995, hereafter RZ95). This makes it possible to build a closure model with plumes (CMP), which is valid in the solar quasi-adiabatic convective region. In a companion paper (Belkacem et al. 2006, hereafter Paper II), we generalize this one-point correlation model to a two-points correlation model and calculate the power injected into solar $p$ modes.

The paper is organised as follows: Sect. 2 introduces the TFM. Its validity is then tested with a $3 \mathrm{D}$ numerical simulation of the uppermost part of the solar convection region. In Sect. 3, we extend the TFM formulation (GTFM) in order to take into account turbulent properties of both upward and downward flows. We next investigate the asymptotic limits of the GTFM. In Sect. 4, we construct the CMP with the help of the RZ95 plume model. We test the validity of this model with results from the $3 \mathrm{D}$ simulation and show that the use of the plume model limits the validity of the CMP to the quasi-adiabatic zone. The CMP is then used to obtain analytical expressions for the third- and fourth-order moments. Section 5 is dedicated to discussions and conclusions.

\section{The two-scale mass-flux model}

\subsection{The model}

The TFM considers a convective medium composed of upward and downward flows that are horizontally averaged. The presence of two flows introduces the possibility of a non-zero skewness for the moments of turbulent quantities when averages are done globally over the whole system. The TFM was developed in order to take into account this non-zero skewness.

Any averaged turbulent quantity $\phi$ can be split into two parts, one associated with the updrafts and the other with the downdrafts:

$\langle\phi\rangle=a\langle\phi\rangle_{\mathrm{u}}+(1-a)\langle\phi\rangle_{\mathrm{d}}$,

where \langle\rangle denotes ensemble spatial (in the horizontal plane) and time averages. $\langle\phi\rangle_{\mathrm{u}}$ and $\langle\phi\rangle_{\mathrm{d}}$ are the averages for the upflow and downflow, respectively. $a$ and $1-a$ are the mean fractional area occupied by the updrafts and downdrafts, respectively (Randall et al. 1992; Gryanik \& Hartmann 2002; Canuto \& Dubovikov 1998).

Fluctuating quantities defined as $\phi^{\prime}=\phi-\langle\phi\rangle$ can be rigourously written as: $\left\langle\phi^{\prime}\right\rangle=a\left\langle\phi^{\prime}\right\rangle_{\mathrm{u}}+(1-a)\left\langle\phi^{\prime}\right\rangle_{\mathrm{d}}$, where the subscripts $u$ and $d$ are meant for upflow and downflow, respectively. For vertical velocity fluctuations $w^{\prime}$, one then writes:

$\left\langle w^{\prime}\right\rangle=a\left\langle w^{\prime}\right\rangle_{\mathrm{u}}+(1-a)\left\langle w^{\prime}\right\rangle_{\mathrm{d}}$

GH2002 propose to make the same decomposition for temperature fluctuations $\left(\theta^{\prime}\right)$; thus, hot and cold regions are considered separately. This step was motivated by the observation that for the case of atmospheric boundary layer the characteristic horizontal scales of velocity and temperature flucutations are different from each other and by the fact that the plain mass flux average Eq. (1) violates certain symmetries between velocity and temperature flucutations. Indeed, hot and cold regions do not necessarily coincide with updrafts and downdrafts, respectively. Hence, a second quantity $(b)$, the mean fractional area occupied by warm drafts, is introduced, and in most cases, $a \neq b$ (thus the name TFM). Then,

$\left\langle\theta^{\prime}\right\rangle=b\left\langle\theta^{\prime}\right\rangle_{\mathrm{h}}+(1-b)\left\langle\theta^{\prime}\right\rangle_{\mathrm{c}}$

Furthermore, the TFM defines the velocity fluctuations inside the upflow $\left(w_{\mathrm{u}}^{\prime}\right)$ and downflow $\left(w_{\mathrm{d}}^{\prime}\right)$, respectively, as:

$w_{\mathrm{u}}^{\prime}=w_{\mathrm{u}}-\langle w\rangle \quad$ and $\quad w_{\mathrm{d}}^{\prime}=w_{\mathrm{d}}-\langle w\rangle$

Similarly, for the temperature fluctuations inside hot $\left(\theta_{\mathrm{h}}^{\prime}\right)$ and cold $\left(\theta_{\mathrm{d}}^{\prime}\right)$ regions, respectively, one has

$\theta_{\mathrm{h}}^{\prime}=\theta_{\mathrm{h}}-\langle\theta\rangle$ and $\theta_{\mathrm{c}}^{\prime}=\theta_{\mathrm{c}}-\langle\theta\rangle$.

The quantities $w_{\mathrm{u}}, w_{\mathrm{d}}, \theta_{\mathrm{h}}$, and $\theta_{\mathrm{c}}$ are the averages of velocity and temperature, respectively, over all updrafts $\left(w_{\mathrm{u}}\right)$, downdrafts $\left(w_{\mathrm{d}}\right)$, hot $\left(\theta_{\mathrm{h}}\right)$ drafts, and cold $\left(\theta_{\mathrm{c}}\right)$ drafts. Clearly, averages of the four fluctuating quantities in Eqs. (4) and (5) do not vanish because the average of a quantity over the whole flow differs from the average over one single (up or down, hot or cold) draft.

It is expected that the differences between the updrafts and downdrafts lead to a probability distribution function (PDF) that is no longer symmetric with respect to vanishing velocities and 
temperature differences. As the third-order moments $\left(\left\langle w^{\prime 3}\right\rangle\right.$ and $\left.\left\langle\theta^{\prime 3}\right\rangle\right)$ vanish when the PDF is symmetric, their values provide a measure for the deviation from a symmetric PDF. The skewnesses are defined as:

$S_{w}=\frac{\left\langle w^{\prime 3}\right\rangle}{\left\langle w^{\prime 2}\right\rangle^{3 / 2}} \quad$ and $\quad S_{\theta}=\frac{\left\langle\theta^{\prime 3}\right\rangle}{\left\langle\theta^{\prime 2}\right\rangle^{3 / 2}}$,

respectively, for the vertical velocity and temperature fluctuations. In order to compute expressions for higher order moments in terms of velocity and temperature fluctuations, Eqs. (4) and (5), GH2002 followed Randall et al. (1992), using an additional simplifying approximation, i.e.,

$\left\langle\phi^{n}\right\rangle \approx\langle\phi\rangle^{n}$,

where $\phi=\left\{w_{\mathrm{u}, \mathrm{d}}^{\prime}, \theta_{\mathrm{h}, \mathrm{c}}^{\prime}\right\}$. This approximation neglects the contributions of flucutations within the up- and downdrafts and differences in temperature and velocity between the individual drafts.

Given this approximation and the known second-order moments, the TFM provides third-order moments as follows (see GH2002):

$\left\langle w^{\prime 2} \theta^{\prime}\right\rangle=S_{w}\left\langle w^{\prime 2}\right\rangle^{1 / 2}\left\langle w^{\prime} \theta^{\prime}\right\rangle$

$\left\langle w^{\prime} \theta^{\prime 2}\right\rangle=S_{\theta}\left\langle\theta^{\prime 2}\right\rangle^{1 / 2}\left\langle w^{\prime} \theta^{\prime}\right\rangle$

and FOMs as:

$$
\begin{aligned}
& \left\langle w^{\prime 4}\right\rangle=\left(1+S_{w}^{2}\right)\left\langle w^{\prime 2}\right\rangle^{2} \\
& \left\langle\theta^{\prime 4}\right\rangle=\left(1+S_{\theta}^{2}\right)\left\langle\theta^{\prime 2}\right\rangle^{2} \\
& \left\langle w^{\prime 3} \theta^{\prime}\right\rangle=\left(1+S_{w}^{2}\right)\left\langle w^{\prime 2}\right\rangle\left\langle w^{\prime} \theta^{\prime}\right\rangle \\
& \left\langle w^{\prime} \theta^{\prime 3}\right\rangle=\left(1+S_{\theta}^{2}\right)\left\langle\theta^{\prime 2}\right\rangle\left\langle w^{\prime} \theta^{\prime}\right\rangle .
\end{aligned}
$$

The skewnesses $S_{w}$ and $S_{\theta}$ (Eq. (6)) are related to $a$ and $b$ through

$S_{w}=\frac{1-2 a}{\sqrt{a(1-a)}} \quad$ and $\quad S_{\theta}=\frac{1-2 b}{\sqrt{b(1-b)}}$

(GH2002; see also Randall et al. 1992, for the case of $S_{w}$ ).

In the following we consider only vertical-velocity moments. Assuming $S_{w}=S_{\theta}=0$ in Eq. (9) gives:

$\left\langle w^{\prime 4}\right\rangle=\left\langle w^{\prime 2}\right\rangle^{2}$.

Such a result is not consistent with a quasi-normal (Gaussian) PDF. Indeed, when $w^{\prime}$ follows a normal distribution, then (Lesieur 1997):

$S_{w}=S_{\theta}=0$ and $\left\langle w^{\prime 4}\right\rangle=3\left\langle w^{\prime 2}\right\rangle^{2}$.

GH2002 found that the two-scale mass-flux average, Eqs. (8)-(10), underestimates both skewness and fourthorder moments as measured by aircraft data for planetary boundary layer convection (see their Figs. 4 and 7). To account for the omitted contributions from fluctuations within and between the up- and downdrafts, they suggested generalizing the TFM by building the fourth-order moments as an interpolation between two asymptotic regimes:

- $S_{w}=0$, assuming the quasi-normal approximation (QNA) limit that is valid for a Gaussian PDF, and

$-S_{w} \gg 1$, the large skewness limit (GH2002).
GH2002 hence proposed:

$$
\begin{aligned}
& \left\langle w^{\prime 4}\right\rangle=3\left(1+\frac{1}{3} S_{w}^{2}\right)\left\langle w^{\prime 2}\right\rangle^{2} \\
& \left\langle\theta^{\prime 4}\right\rangle=3\left(1+\frac{1}{3} S_{\theta}^{2}\right)\left\langle\theta^{\prime 2}\right\rangle^{2} \\
& \left\langle w^{\prime 3} \theta^{\prime}\right\rangle=3\left(1+\frac{1}{3} S_{w}^{2}\right)\left\langle w^{\prime 2}\right\rangle\left\langle w^{\prime} \theta^{\prime}\right\rangle \\
& \left\langle w^{\prime} \theta^{\prime 3}\right\rangle=3\left(1+\frac{1}{3} S_{\theta}^{2}\right)\left\langle\theta^{\prime 2}\right\rangle\left\langle w^{\prime} \theta^{\prime}\right\rangle
\end{aligned}
$$

Corresponding expressions for other FOMs $\left(\left\langle w^{\prime 2} \theta^{2}\right\rangle\right.$ and those including horizontal velocities) can be found in Gryanik et al. (2005, hereafter GH2005).

\subsection{Validation with a $3 D$ numerical simulation of the solar external layers}

We consider the uppermost part of the solar turbulent convection. Turbulent plumes are known to exist within this region (Cattaneo et al. 1991; Stein \& Nordlund 1998). Here, we test the validity of the TFM using 3D numerical simulations of these upper solar layers. The geometry is plane-parallel with a physical size of $6 \mathrm{Mm} \times 6 \mathrm{Mm} \times 3 \mathrm{Mm}$. The upper boundary corresponds to a convectively stable atmosphere and the lower one to a quasi-adiabatic convection zone. The 3D simulations used in this work were obtained with Stein \& Nordlund's 3D numerical code (Stein \& Nordlund 1998). Two simulations with different spatial grids were considered: $253 \times 253 \times 163$ and $125 \times 125 \times 82$.

Averages and moments of the velocity and temperature fluctuations were computed in a two-stage process:

$a$ is given as the number of grid points per layer with upwards directed vertical velocity divided by the total number of points in that layer. The instantaneous value of $b$ is obtained in a similar manner, comparing the temperature at a given point in a layer with its horizontal average. Moments related to updrafts were obtained from horizontal averaging, using only those grid points at which vertical velocity was directed upwards at the given instant in time, and likewise, quantities related to downdrafts were obtained from horizontal averaging using only those grid points at which vertical velocity was directed downwards. In a second step, time averages were performed over a sufficiently long period of time such that averages no longer depended on the integration time beyond a few percent.

- Calculation of the skewnesses: computations of the mean fractional area of the upflow $(a)$ and downflow $(1-a)$, as well those of the warm $(b)$ and cold $(1-b)$ drafts from the numerical 3D simulations (Fig. 1), show that the upper part of the solar convection zone can be divided into three parts: the stable atmosphere, the superadiabatic zone, and the quasi-adiabatic zone. In the convectively stable atmosphere $(z<0 \mathrm{Mm}$, where $z=0$ is approximately at the bottom of the photosphere and $z=-0.5 \mathrm{Mm}$ is the uppermost boundary of the simulation), there are no asymmetric motions. In the superadiabatic zone $(0<z<0.5 \mathrm{Mm})$, from the top downwards, the departure from symmetry for the flows strongly increases (Fig. 1), and the skewnesses, $S_{w}$ and $S_{\theta}$, significantly differ from zero (Fig. 2). Hence, one must expect a non-negligible departure from the QNA, which is explained by radiative cooling creating turbulent plumes. In the quasi-adiabatic zone, plumes have already been formed and no additional asymmetry is therefore created. Hence, 

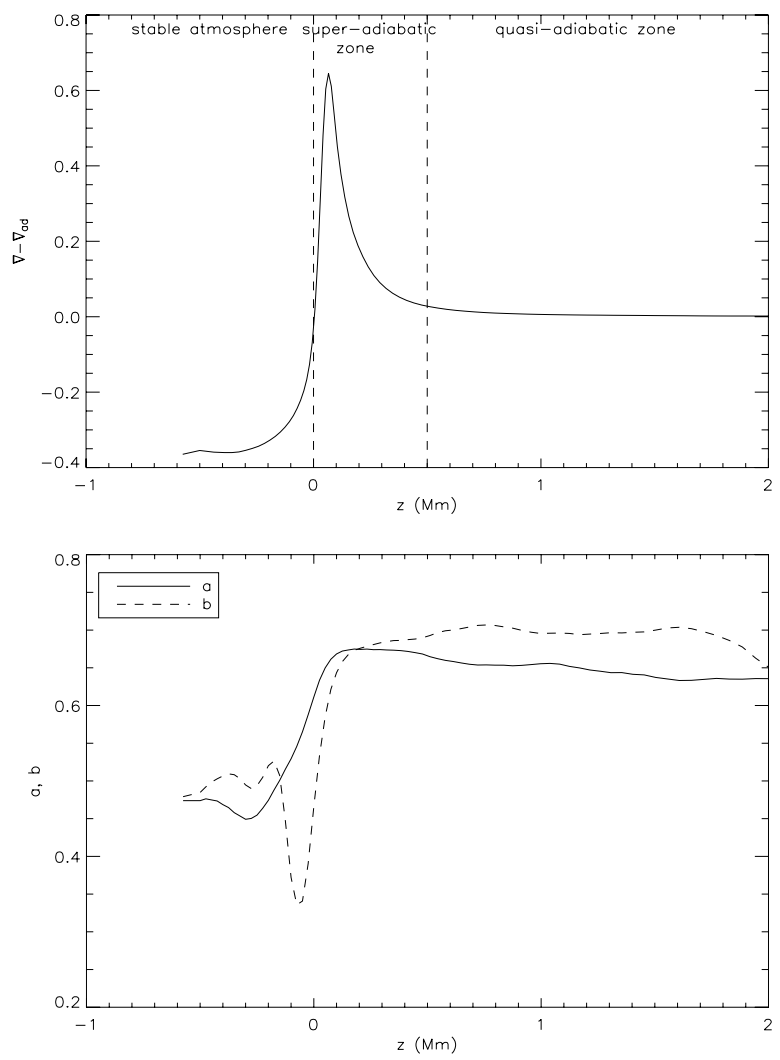

Fig. 1. On the top, the superadiabatic gradient $\left(\nabla-\nabla_{\mathrm{ad}}\right)$ is plotted versus the depth $(z)$. The reference depth $(z=0 \mathrm{Mm})$ corresponds to the photosphere. At the bottom, the mean fractional area of the upflow $(a)$ and the warm drafts $(b)$ are given. To calculate these quantities the upflow and downflow are separated using the sign of $w^{\prime}$ as a criterion. The same is done for the warm and cold drafts.

the asymmetry remains large and constant $(a \approx b \approx 0.7)$ and the skewnesses show a constant departure from $S_{w}=S_{\theta}=0$.

The last two regions are of interest in this work because both show a departure from the quasi-normal PDF in terms of fluctuating vertical velocity and temperature. The comparison of the above numerical results with the results from the classical TFM (Eq. (9)) and the TFM model (Fig. 2) shows that Eq. (10) fails to reproduce the behaviour of the skewnesses from the 3D simulation (as was also found by Gryanik \& Hartmann 2002 for convection in the atmosphere of the Earth, see their Fig. 4).

- Detailed comparison of a fourth-order moment: the GH2002 interpolation relation Eq. (14) combined with the TFM relation for skewness, Eq. (10), shows only a slight improvement of the QNA description for the FOM $\left\langle w^{\prime 4}\right\rangle$, when compared to the numerical result (Fig. 3).

To conclude, it seems that a physical process is missing in the quasi-adiabatic convective zone. To explain such a disagreement between the numerical results and the TFM, we must come back to its main approximation (see Eq. (7)). For $n=2$, Eq. (7) yields:

$\left\langle w^{\prime 2}\right\rangle-\left\langle w^{\prime}\right\rangle^{2} \approx 0 \quad\left\langle\theta^{\prime 2}\right\rangle-\left\langle\theta^{\prime}\right\rangle^{2} \approx 0$.

Hence, the TFM assumes that the variances of the fluctuations of vertical velocity and temperature within and among individual drafts vanish, and the detailed turbulent nature of the flows themselves does not have to be taken into account. In order to compensate for the shortcoming of Eq. (9) and thus the consequences of the approximation Eq. (7) on the model predictions, Gryanik \& Hartmann (2002) proposed a more general interpolation
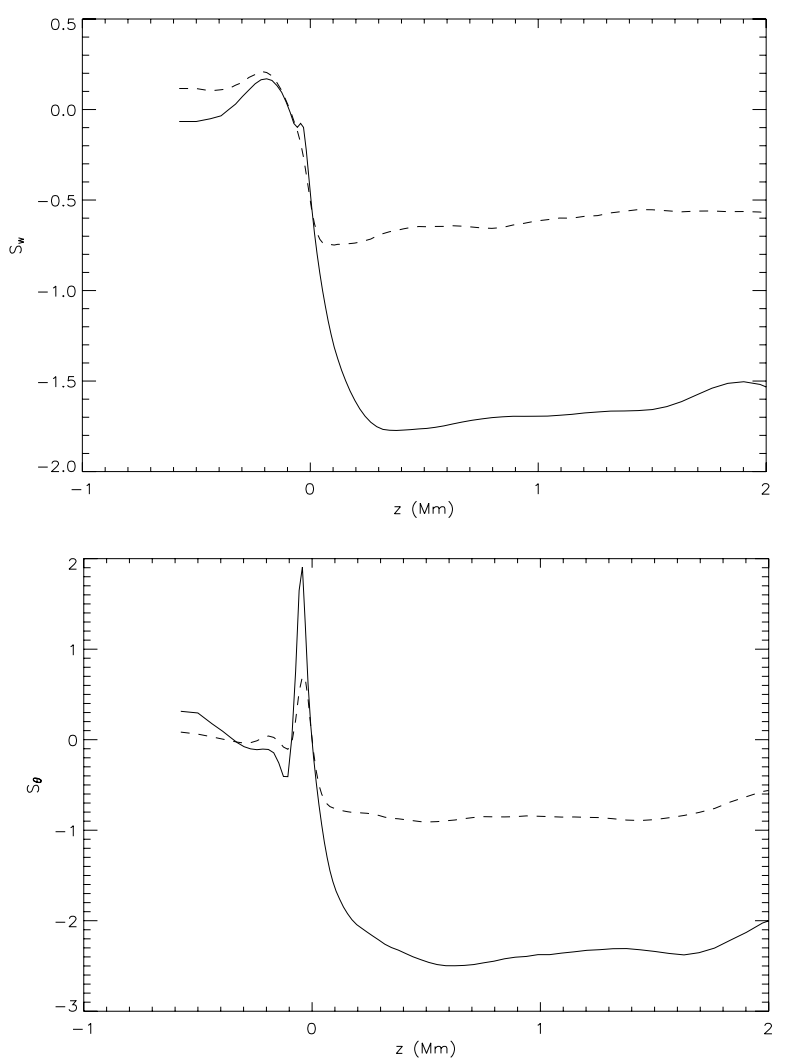

Fig. 2. The skewnesses $S_{w}$ (on the top) and $S_{\theta}$ (on the bottom) are plotted versus the depth $(z)$. Solid lines represent direct calculation from the 3D numerical simulation (Eq. (6)) and dashed lines represent the skewnesses calculated using the TFM model (Eq. (10)).

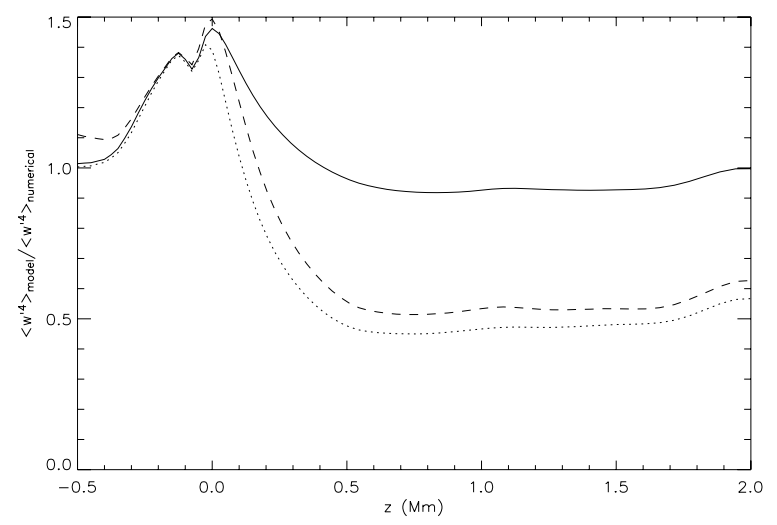

Fig. 3. Fourth-order moment $\left(\left\langle w^{\prime 4}\right\rangle\right)$ as a function of depth $(z)$ normalized to the FOM, as calculated directly from the simulation. The solid line denotes the moment calculated using Eq. (14) with $S_{w}$ taken directly from the simulation; the dashed line shows the result if $S_{w}$ is instead taken from Eq. (10), as in the TFM case; and the dotted line is the QNA (Eq. (26)). Equations (14) and (26) involve second-order moments that are computed using the numerical simulation.

relation (Eq. (14)) that uses Eq. (9) only for one of two asymptotic limits.

As seen above, Eq. (10) fails to describe the numerical results. The question therefore is whether the interpolated relation (Eq. (14)) is still valid, provided a correct value for the skewness is used. Hence, we assess the validity of Eq. (14) by inserting the value of $S_{w}$ directly given by the 3D numerical result. The result is shown in Fig. 3 as well. This is the model that Gryanik \& Hartmann (2002) proposed to be used instead of the TFM 
itself and its associated relation for the skewnesses, Eq. (10). We obtain an accurate description of the FOM $\left\langle w^{\prime 4}\right\rangle$ in the quasiadiabatic region, but not in the superadiabatic zone, where the interpolated relation does not seem well adapted (cf. KR2006 for a more detailed discussion).

\section{The generalized two-scale mass-flux model}

\subsection{Theoretical formulation}

Here we remove the approximation of Eq. (7) and instead consider the exact expression:

$\left\langle w^{\prime n}\right\rangle=a\left\langle w^{\prime n}\right\rangle_{\mathrm{u}}+(1-a)\left\langle w^{\prime n}\right\rangle_{\mathrm{d}}$.

Our main idea is to separate the effect of the skewness introduced by the presence of two flows from the effect of the turbulence that occurs in each individual flow. We note that in a geophysical context Siebesma \& Cuijpers (1995) and Petersen et al. (1999) studied the transport properties of classical mass-flux models that also involved a separation of large-scale and turbulent components. Here, we start from the more recent viewpoint of the TFM by Gryanik \& Hartmann (2002) and Gryanik et al. (2005), which takes into account that updrafts and downdrafts are not strictly correlated with hot and cold drafts, respectively. As a first step we define the intrinsic fluctuations within one of the flows as:

$\tilde{w}_{j}^{\prime}=w_{j}-\langle w\rangle_{j}$,

where $j=\{\mathrm{u}, \mathrm{d}\}$. They are fluctuations with vanishing averages. To express $w_{j}^{\prime}$ in terms of $\tilde{w}_{j}^{\prime}$ (Eq. (4)), we write:

$w_{j}^{\prime}=\tilde{w}_{j}^{\prime}+\langle w\rangle_{j}-\langle w\rangle$.

Applying the decomposition of Eq. (1) to $\langle w\rangle$ in the above expression yields:

$w_{\mathrm{u}}^{\prime}=\tilde{w}_{\mathrm{u}}^{\prime}+(1-a) \delta w$

$w_{\mathrm{d}}^{\prime}=\tilde{w}_{\mathrm{d}}^{\prime}-a \delta w$

with

$\delta w=\langle w\rangle_{\mathrm{u}}-\langle w\rangle_{\mathrm{d}}=\left|\langle w\rangle_{\mathrm{u}}\right|+\left|\langle w\rangle_{\mathrm{d}}\right|$,

because $\langle w\rangle_{\mathrm{u}}>0$ and $\langle w\rangle_{\mathrm{d}}<0$.

Inserting Eq. (18) into Eq. (15) for $n=2,3$ yields:

$$
\begin{aligned}
\left\langle w^{\prime 2}\right\rangle= & a(1-a) \delta w^{2}+a\left\langle\tilde{w}^{2}\right\rangle_{\mathrm{u}}+(1-a)\left\langle\tilde{w}^{\prime 2}\right\rangle_{\mathrm{d}} \\
\left\langle w^{\prime 3}\right\rangle= & a(1-a)(1-2 a) \delta w^{3}+a\left\langle\tilde{w}^{\prime 3}\right\rangle_{\mathrm{u}}+(1-a)\left\langle\tilde{w}^{\prime 3}\right\rangle_{\mathrm{d}} \\
& +3 a(1-a)\left[\left\langle\tilde{w}^{\prime 2}\right\rangle_{\mathrm{u}}-\left\langle\tilde{w}^{\prime 2}\right\rangle_{\mathrm{d}}\right] \delta w .
\end{aligned}
$$

The third-order moment (Eq. (21)), which is related to the skewness (see Eq. (6)), is composed of four contributions:

- the first term is the expression derived by Gryanik \& Hartmann (2002). It is a measure of the skewness introduced by the presence of two flows;

- the second and third terms represent the asymmetry of the PDF within each flow induced by turbulence;

- the fourth term measures the difference of the fluctuating velocity dispersion. Hence, if one of them is larger than the other, the PDF becomes asymmetric.
The description of the turbulence in individual flows that has been neglected in the TFM is included in the present formulation through the last three terms in Eq. (21).

We next focus on the fourth-order moment $\left\langle w^{\prime 4}\right\rangle$, which is of interest in the context of stochastic excitation of solar $p$ modes (see Paper II). Then setting $n=4$ in Eq. (15), we have:

$$
\begin{aligned}
\left\langle w^{\prime 4}\right\rangle= & a(1-a)\left(1-3 a+3 a^{2}\right) \delta w^{4} \\
& +6 a(1-a)\left((1-a)\left\langle\tilde{w}^{\prime 2}\right\rangle_{\mathrm{u}}+a\left\langle\tilde{w}^{\prime 2}\right\rangle_{\mathrm{d}}\right) \delta w^{2} \\
& +4 a(1-a)\left(\left\langle\tilde{w}^{\prime 3}\right\rangle_{\mathrm{u}}-\left\langle\tilde{w}^{\prime 3}\right\rangle_{\mathrm{d}}\right) \delta w \\
& +a\left\langle\tilde{w}^{\prime 4}\right\rangle_{\mathrm{u}}+(1-a)\left\langle\tilde{w}^{\prime 4}\right\rangle_{\mathrm{d}} .
\end{aligned}
$$

We stress that the TFM is recovered from the present generalized formulation when proper fluctuations (i.e., turbulence) within and among the individual drafts are neglected, i.e., $\left\langle\tilde{w}^{\prime \prime}\right\rangle=0$.

The same decomposition can be performed in terms of temperature fluctuations. As the calculation is symmetrical in $w^{\prime}, a$ and $\theta^{\prime}, b$, we hence have:

$$
\begin{aligned}
\left\langle\theta^{\prime 2}\right\rangle= & b(1-b) \delta \theta^{2} \\
& +b\left\langle\tilde{\theta}^{\prime 2}\right\rangle_{\mathrm{h}}+(1-b)\left\langle\tilde{\theta}^{\prime 2}\right\rangle_{\mathrm{c}} \\
\left\langle\theta^{\prime 3}\right\rangle= & b(1-b)(1-2 b) \delta \theta^{3} \\
& +b\left\langle\tilde{\theta}^{\prime 3}\right\rangle_{\mathrm{h}}+(1-b)\left\langle\tilde{\theta}^{\prime 3}\right\rangle_{\mathrm{c}} \\
& +3 b(1-b)\left[\left\langle\tilde{\theta}^{\prime 2}\right\rangle_{\mathrm{h}}-\left\langle\tilde{\theta}^{\prime 2}\right\rangle_{\mathrm{c}}\right] \delta \theta \\
\left\langle\theta^{\prime 4}\right\rangle= & b(1-b)\left(1-3 b+3 b^{2}\right) \delta \theta^{4} \\
& +6 b(1-b)\left((1-b)\left\langle\tilde{\theta}^{\prime 3}\right\rangle_{\mathrm{h}}+b\left\langle\tilde{\theta}^{\prime 2}\right\rangle_{\mathrm{c}}\right) \delta \theta^{2} \\
& +4 b(1-b)\left(\left\langle\tilde{\theta}^{\prime 3}\right\rangle_{\mathrm{h}}-\left\langle\tilde{\theta}^{\prime 3}\right\rangle_{\mathrm{c}}\right) \delta \theta \\
& +b\left\langle\tilde{\theta}^{\prime 4}\right\rangle_{\mathrm{h}}+(1-b)\left\langle\tilde{\theta}^{\prime 4}\right\rangle_{\mathrm{c}}
\end{aligned}
$$

The next step consists of the derivation of the cross terms $\left\langle w^{\prime} \theta^{\prime}\right\rangle,\left\langle w^{\prime 2} \theta^{\prime 2}\right\rangle,\left\langle w^{\prime 2} \theta^{\prime}\right\rangle$ and $\left\langle w^{\prime} \theta^{\prime 2}\right\rangle$; it is convenient to define the coefficients $a_{\mathrm{uh}}, a_{\mathrm{uc}}$ so as to take into account the four types of flow (see also GH2005):

- warm updraft, $a_{\mathrm{uh}}$

- cold updraft, $a_{\mathrm{uc}}=a-a_{\mathrm{uh}}$

- warm downdraft, $a_{\mathrm{dh}}=b-a_{\mathrm{uh}}$

- cold downdraft, $a_{\mathrm{dc}}=1-b-a_{\mathrm{uc}}$.

Expressions for the third and fourth cross-correlation moments are given in Appendix A.

The generalized TFM has the advantage of isolating the skewness introduced by the two flows (as measured by $S_{w}$ and $S_{\theta}$ in Eq. (10)) from the effects of turbulence in each of the flows (as measured for instance by the two terms $\tilde{w}_{\mathrm{d}}^{\prime 2}$ and $\tilde{w}_{\mathrm{u}}^{\prime 2}$ ). The GTFM allows us to take the effects of turbulence into account. We note that a small value of the kurtosis can occur only if proper fluctuations lead to negligibly small deviations from the root mean square average. Such a flow pattern consisting of clearly defined up- and downflows as well as hot and cold areas with a kurtosis $K_{w} \gtrsim 1$ can be considered as representing a quasi-laminar state. We stress that for the quasi-laminar case, Eq. (9) remains exact; thus the kurtosis becomes:

$K_{w}=\frac{\left\langle w^{\prime 4}\right\rangle}{\left\langle w^{\prime 2}\right\rangle^{2}}=\left(1+S_{w}^{2}\right)$ with $S_{w}=\frac{1-2 a}{\sqrt{a(1-a)}}$.

For $a=0.5$, one obtains $K_{w}=1$, which is far from the value for a Gaussian PDF $\left(K_{w}=3\right)$. To take into account turbulence within 
the up- and downdrafts, one can use Eq. (14) (see Sect. 2.2) with the skewness $S_{w}=\left\langle w^{\prime 3}\right\rangle /\left\langle w^{\prime 2}\right\rangle^{3 / 2}$ from the GTFM. In this case we obtain:

$K_{w}=3\left(1+\frac{1}{3} S_{w}^{2}\right)$

This implies that a (moderately small) non-vanishing skewness will make the value of $K_{w}$ closer to three than in the quasilaminar case. In the solar case, in the quasi-isentropic zone $S_{w}^{2} \approx 4$ (Fig. 2), hence $K_{w} \approx 3+4 / 3$. In the physical picture underlying Eq. (14), turbulence prevents the PDF from being too far from a Gaussian one $\left(K_{w} \rightarrow 3\right)$.

We notice that one important source of turbulence that can be considered responsible for at least part of the fluctuations in a draft - in addition to those created by the radiative processes on top of the convection zone - is related to shearing stresses between the up- and downdrafts. However, the investigation of the sources of turbulence is beyond the scope of the present work. Those mechanisms certainly play an important role in both the small scale velocity and the thermal fluctuations. Their study is definitely desirable in the future. One should also note that the splitting approach of the GTFM is valid and can be used for any convective system, provided that it is composed of two flows. As it is unclosed, it must be seen as a good basis for building a closure model.

\subsection{Asymptotic limits}

In the following, we study the asymptotic limits of the GTFM, focusing on the fourth-order moment $\left\langle w^{\prime 4}\right\rangle$. The standard mass flux model is easily recovered when setting the proper moments to zero: $\left\langle\tilde{w}^{\prime n}\right\rangle=0$ in Eqs. (20)-(22). The same holds for the TFM, Eqs. (8)-(9), which is recovered, if in addition $\left\langle\tilde{\theta}^{\prime \prime}\right\rangle=0$ in Eq. (23) (cf. Eqs. (7) and (8) in Gryanik \& Hartmann 2002). We now turn to the QNA limit and the limit for large skewness, which are more interesting as they are used by Gryanik \& Hartmann (2002) and Gryanik et al. (2005) in order to corroborate the interpolation formula Eq. (14).

\subsubsection{The quasi-normal limit}

To obtain the QNA (Eq. (12)), it is necessary that $S_{w}=0$, but it is not sufficient. In fact, a vanishing skewness only shows that the PDF is symmetric, but not that the PDF is Gaussian. Further conditions are necessary:

- the moments must have zero mean, which implies $\left|\langle w\rangle_{\mathrm{u}}\right|=$ $\left|\langle w\rangle_{\mathrm{d}}\right|=0$ from Eqs. (2) and (18);

- for the QNA to apply to the whole system, one must assume that the QNA is valid for each flow;

- we must also assume that $a=0.5$;

- the turbulent pressure must be the same in the upflow and downflow. Otherwise the skewness $\left(S_{w}\right)$ is different from zero, according to Eq. (21), and the consequence is an asymmetric PDF, which is not consistent with the quasi-normal assumption. So the condition $\left\langle\tilde{w}^{\prime 2}\right\rangle_{\mathrm{u}}=\left\langle\tilde{w}^{\prime 2}\right\rangle_{\mathrm{d}}$ is required.

Then starting with Eq. (22), we find:

$$
\begin{aligned}
\left\langle w^{\prime 4}\right\rangle & =a\left\langle\tilde{w}^{\prime 4}\right\rangle_{\mathrm{u}}+(1-a)\left\langle\tilde{w}^{\prime 4}\right\rangle_{\mathrm{d}} \\
& =\frac{3}{2}\left\langle\tilde{w}^{\prime 2}\right\rangle_{\mathrm{u}}^{2}+\frac{3}{2}\left\langle\tilde{w}^{\prime 2}\right\rangle_{\mathrm{d}}^{2},
\end{aligned}
$$

and finally

$\left\langle w^{4}\right\rangle=3\left\langle w^{\prime 2}\right\rangle^{2}$, which is the expression for the fourth order moment in the QNA. Note that the TFM (Sect. 2) is unable to properly recover the QNA. Within the GTFM the QNA results from two terms, $\left\langle\tilde{w}^{\prime 4}\right\rangle_{\mathrm{u}}$ and $\left\langle\tilde{w}^{\prime 4}\right\rangle_{\mathrm{d}}$, which are related to the intrinsic turbulence in each flow, but these are neglected in the TFM. This example also demonstrates that for a convective flow, the deviation of a PDF from a Gaussian one cannot be modelled by the TFM without further modifications of that model (even if $a=0.5$ ).

\subsubsection{The large skewness limit}

Gryanik et al. (2005) have shown that the TFM must be recovered when considering a convective system with large skewness. Then, for $S_{w} \gg 1$, the expression for $\left\langle w^{\prime 4}\right\rangle$ in Eq. (14) becomes: $\left\langle w^{\prime 4}\right\rangle \approx S_{w}^{2}\left\langle w^{\prime 2}\right\rangle^{2}$.

The large skewness limit physically corresponds to either $a \approx 1$ or $a \approx 0$. Indeed, it means that one of the two flows dominates over the other one in terms of mean fractional area in the horizontal plane. Thus, due to conservation of mass, the mean vertical velocity becomes large such that $\delta w \gg 1 \mathrm{~m} \mathrm{~s}^{-1}$ in the solar case (see Sect. 4, Eq. (41)).

In Eq. (22), the term proportional to $\delta w^{4}$, which measures the effects introduced by an asymmetric flow, dominates and leads to the TFM expression for the fourth-order moment $\left\langle w^{\prime 4}\right\rangle$ :

$\left\langle w^{4}\right\rangle=a(1-a)\left(a^{3}+(1-a)^{3}\right) \delta w^{4}$.

Gryanik \& Hartmann (2002) demonstrated that this expression leads to the relation:

$\left\langle w^{\prime 4}\right\rangle=\left(1+S^{2}\right)\left\langle w^{\prime 2}\right\rangle^{2} \approx S^{2}\left\langle w^{\prime 2}\right\rangle^{2}$ for $S \gg 1$,

where, as in Eq. (10), $S=(1-2 a) / \sqrt{a(1-a)}$. The same would result if the exact function $S_{w}$ were taken in this limit instead of its approximation, Eq. (10).

Hence, the GTFM enables us to show that the asymptotic limits used by Gryanik \& Hartmann (2002) to motivate the interpolated expressions for the FOMs (Eq. (14)) are limiting cases for a flow that consists of a coherent part with two components (up- and downdrafts), which themselves are subject to turbulence (cf. the discussion of the GH2002 model in KR2006). In Sect. 2.2 we have shown, using the 3D numerical simulation, that this interpolation is valid provided the skewness is taken directly from the $3 \mathrm{D}$ simulation. This property can be understood using the GTFM, as it permits us to obtain the different ingredients of the interpolation formula of Gryanik \& Hartmann (2002) from Eq. (22) and the individual contributions to Eq. (22), can be analyzed using numerical simulations.

\section{The closure model with plumes}

Section 2.2 confirmed the conclusion by KR2006 that the interpolated relations in Eq. (14) proposed by Gryanik \& Hartmann (2002) could be adapted for the solar case provided that the skewnesses are appropriately calculated. Using the GTFM to model skewnesses, Eq. (21) shows that the skewness $S_{w}$, for instance, depends on six quantities: $\delta w,\left\langle\tilde{w}^{\prime 3}\right\rangle_{\mathrm{u}, \mathrm{d}},\left\langle\tilde{w}^{\prime 2}\right\rangle_{\mathrm{u}, \mathrm{d}}$, and $a$. As shown below, some of the terms in $S_{w}$ turn out to be negligible in the quasi-adiabatic convective region because plumes are more turbulent in the downflow than in the upflow (Stein \& Nordlund 1998). The remaining dominant terms are modelled hereafter by a plume model (Rieutord \& Zahn 1995) in the quasi-adiabatic convective region, where the CMP is valid. 


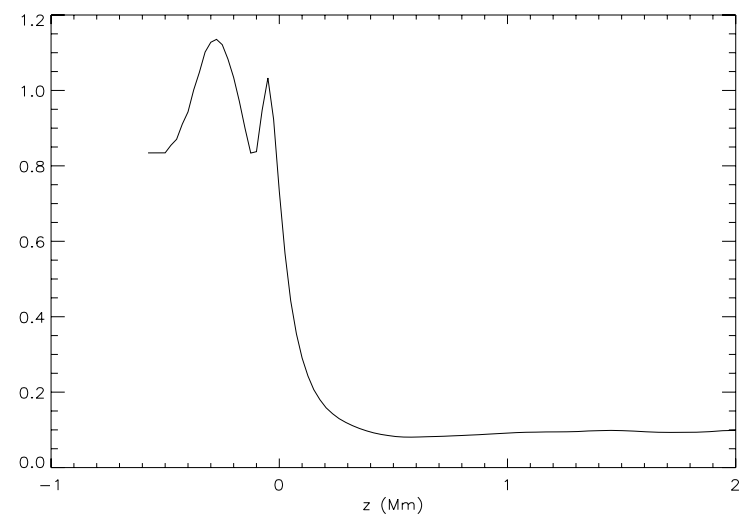

Fig. 4. Second-order moment of the upflow over that of the downflow $\left(\left\langle\tilde{w}^{\prime 2}\right\rangle_{\mathrm{u}} /\left\langle\tilde{w}^{\prime 2}\right\rangle_{\mathrm{d}}\right)$ as a function of depth, calculated directly from the simulation. Upflow and downflow are determined according to the sign of $w^{\prime}$.

\subsection{Turbulence in upflows and downflows}

In Fig. 4, we compare the second-order moments of both flows. These quantities are of the same order of magnitude in the upper part, above the photosphere. From the photosphere, the ratio $\left\langle\tilde{w}^{\prime 2}\right\rangle_{\mathrm{u}} /\left\langle\tilde{w}^{\prime 2}\right\rangle_{\mathrm{d}}$ then sharply decreases, with increasing depth $(z)$. Hence, contributions to the skewness $\left(S_{w}\right)$, involving $\left\langle\tilde{w}^{\prime 2}\right\rangle_{\mathrm{u}}$ (Eqs. (20) and (21)) can be neglected in comparison with those involving $\left\langle\tilde{w}^{\prime 2}\right\rangle_{\mathrm{d}}$ in the quasi-adiabatic part of the convection zone. The third-order moments $\left\langle\tilde{w}^{\prime 3}\right\rangle_{\mathrm{d}}$ and $\left\langle\tilde{w}^{\prime 3}\right\rangle_{\mathrm{u}}$ can also be discarded (see Fig. 5) because their contributions are negligible.

The skewness $S_{w}$ then becomes:

$S_{w}=\frac{a(1-a)}{\left\langle w^{\prime 2}\right\rangle^{3 / 2}}\left((1-2 a) \delta w^{2}-3\left\langle\tilde{w}^{\prime 2}\right\rangle_{\mathrm{d}}\right) \delta w$,

where $\delta w$ is given by Eq. (19). Hence, only $\left\langle\tilde{w}^{\prime 2}\right\rangle_{\mathrm{d}}$ and $\delta w$ remain to be modelled. Similarly, the 3D calculations show that the cool medium is more turbulent than the hot one and that third-order moments for the temperature fluctuations can be neglected. Then the expression for $S_{\theta}$ becomes:

$S_{\theta}=b(1-b) \frac{1}{\left\langle\theta^{\prime 2}\right\rangle^{3 / 2}}\left((1-2 b) \delta \theta^{2}-3\left\langle\tilde{\theta}^{\prime 2}\right\rangle_{\mathrm{c}}\right) \delta \theta$,

where the quantities $\delta \theta=\langle\theta\rangle_{\mathrm{h}}-\langle\theta\rangle_{\mathrm{c}}$ and $\left\langle\tilde{\theta}^{\prime 2}\right\rangle_{\mathrm{c}}$ must be modelled.

Note that in the QNA limit $\delta w=0$, so that for the expression Eq. (30), $S_{w}=0$, and according to Eq. (14),

$\left\langle w^{\prime 4}\right\rangle=3\left\langle w^{\prime 2}\right\rangle^{2}$.

However, because we have assumed $\left\langle\tilde{w}^{\prime 2}\right\rangle_{\mathrm{u}} \ll\left\langle\tilde{w}^{\prime 2}\right\rangle_{\mathrm{d}}$ when deriving the expression $S_{w}$, rigourously speaking, $S_{w}$ does not tend correctly to zero in the QNA limit. Such an expression therefore cannot be used in the case of a near QNA regime. In our case, we have shown in Sect. 2.2 that the medium is far from the QNA limit in the quasi-adiabatic zone, and hence the expression Eq. (30) can be safely used.

To proceed further, $\left\langle\tilde{w}^{\prime 2}\right\rangle_{\mathrm{d}}$ and $\left\langle\tilde{\theta}^{\prime 2}\right\rangle_{\mathrm{c}}$ are written in a more suitable form. We neglect $\left\langle\tilde{w}^{\prime 2}\right\rangle_{\mathrm{u}}$ in Eq. (20) for $\left\langle w^{\prime 2}\right\rangle$, and $\left\langle\tilde{\theta}^{2}\right\rangle_{\mathrm{h}}$ in Eq. (23) for $\left\langle\theta^{\prime 2}\right\rangle$. This yields:

$\left\langle w^{\prime 2}\right\rangle=a(1-a) \delta w^{2}+(1-a)\left\langle\tilde{w}^{2}\right\rangle_{\mathrm{d}}$

$\left\langle\theta^{\prime 2}\right\rangle=b(1-b) \delta \theta^{2}+(1-b)\left\langle\tilde{\theta}^{\prime 2}\right\rangle_{\mathrm{c}}$.

We then derive expressions for $\left\langle\tilde{w}^{\prime 2}\right\rangle_{\mathrm{d}}$ and $\left\langle\tilde{\theta}^{\prime 2}\right\rangle_{\mathrm{c}}$ in terms of $\left\langle w^{\prime 2}\right\rangle$, $\delta w$, and $\left\langle\theta^{\prime 2}\right\rangle, \delta \theta$, respectively (see Eq. (32)). Inserting them into Eqs. (30) and (31), one then obtains:

$S_{w}=\frac{1}{\left\langle w^{\prime 2}\right\rangle^{3 / 2}} a\left((1-a)(1-5 a) \delta w^{2}-3\left\langle w^{\prime 2}\right\rangle\right) \delta w$

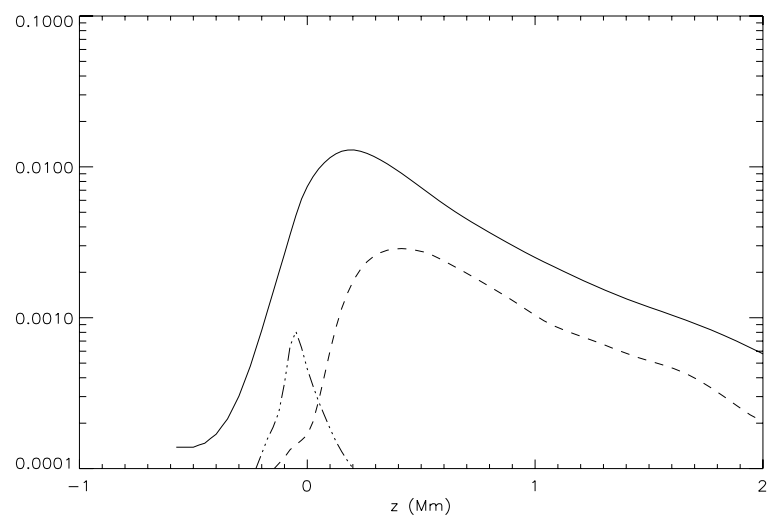

Fig. 5. The terms $3 a(1-a)\left\langle\tilde{w}^{\prime 2}\right\rangle_{\mathrm{d}} \delta w$ (solid line), $a\left\langle\tilde{w}^{\prime 3}\right\rangle_{\mathrm{u}}$ (dot-dot-dotdashed line), and $(1-a)\left\langle\tilde{w}^{\prime 3}\right\rangle_{\mathrm{d}}$ (dashed line) are plotted versus the depth (z). From Eq. (21), the dominant terms remains $3 a(1-a)\left\langle\tilde{w}^{2}\right\rangle_{\mathrm{d}} \delta w$. This justifies the assumptions that the terms involving third-order moments can be neglected in the quasi-adiabatic zone.

and

$S_{\theta}=\frac{1}{\left\langle\theta^{\prime 2}\right\rangle^{3 / 2}} b\left((1-b)(1-5 b) \delta \theta^{2}-3\left\langle\theta^{\prime 2}\right\rangle\right) \delta \theta$.

We assume that the second-order moments $\left(\left\langle w^{2}\right\rangle\right.$ and $\left.\left\langle\theta^{\prime 2}\right\rangle\right)$ are known. In the present work, they are computed from the 3D numerical simulation. In principle, they could also be taken from a convection model such as the mixing-length theory. The last step then is to determine $\delta w$ and $\delta \theta$ (as well as $a$ and $b$ ). As $\delta w$ is the difference between the mean velocities of upward and downward flows, it is possible to model it by means of a plume model. This approach is also used to determine $\delta \theta$.

\subsection{The plume model}

\subsubsection{Determination of $\delta w$}

We use the model of plumes developed by Rieutord \& Zahn (1995). The plume is considered in an axisymmetric geometry with a Gaussian horizontal profile for the vertical velocity $\left(w_{\mathrm{d}}\right)$, the fluctuations of enthalpy $(\delta h)$, and density $(\delta \rho)$ such that

$w_{\mathrm{d}}(r, z)=V(z) \exp \left(-r^{2} / b_{\mathrm{p}}^{2}\right)$,

$\delta \rho(r, z)=\Delta \rho(z) \exp \left(-r^{2} / b_{\mathrm{p}}^{2}\right)$, and

$\delta h(r, z)=\Delta h(z) \exp \left(-r^{2} / b_{\mathrm{p}}^{2}\right)$.

where $b_{\mathrm{p}}(z)$ is the radius of the plume. We assume, as in RZ95, an isentropic and polytropic envelope structure, hence

$\rho(z)=\rho_{0}\left(z / z_{0}\right)^{q}$,

$T(z)=T_{0}\left(z / z_{0}\right)$,

where $q$ is the polytropic coefficient. $\rho_{0}$ and $T_{0}$ are the density and temperature at depth $z=z_{0}$, and $z_{0}$ is the reference depth that corresponds to the base of the convective region.

In Fig. 6, we show that the mean velocity of upflow and downflow in the quasi-adiabatic convection zone both obey a power law in $\left(z / z_{0}\right)^{r}$. We therefore assume a power law for the mean velocity of the downflow (i.e., the plumes). Then

$\langle w\rangle_{\mathrm{d}}=w_{\mathrm{d} 0}\left(\frac{z}{z_{0}}\right)^{r}$

with

$w_{\mathrm{d} 0}=\left(\frac{12 F}{\beta_{0}^{2} \pi \rho_{0} g z_{0}^{2}}\right)^{1 / 3}$, 


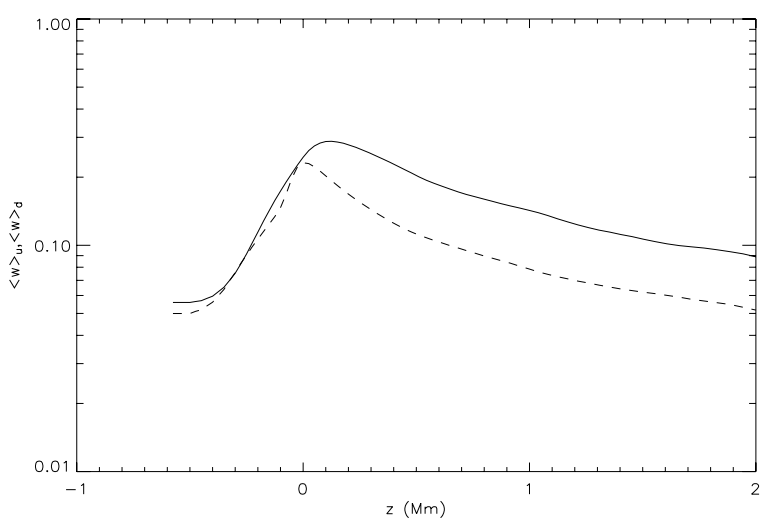

Fig. 6. Mean velocity profile of the upflow (dashed line) and downflow (solid line) as a function of the depth. Note that the peak at $z=0.1 \mathrm{Mm}$ corresponds to the maximum turbulent pressure. The use of power laws limits the validity of the CMP to the quasi-adiabatic zone, as is implied by the deviation of the profiles from power laws in the superadiabatic region.

Table 1. Solar values of plume model parameters (from RZ95).

\begin{tabular}{cc}
\hline \hline$\beta_{0}$ & $\approx 0.1$ \\
$\rho_{0}$ & $190 \mathrm{~kg} \mathrm{~m}^{-3}$ \\
$L_{\odot}$ & $3.9 \times 10^{26} \mathrm{~W}$ \\
$z_{0}$ & $\approx 2 \times 10^{8} \mathrm{~m}$ \\
$g_{\odot}$ & $270 \mathrm{~m} \mathrm{~s}^{-2}$ \\
\hline
\end{tabular}

(RZ95), where $r=(-q+1) / 3, \beta_{0}=3 \alpha /(q+2)$, and $\alpha=0.083$ is the entrainment constant for a Gaussian profile (Turner 1986). $F$ is the convective energy flux and $g$ is the gravitational acceleration. In Table 1, we list solar values of the previously introduced parameters taken from RZ95. These values are used in the present paper except for $F$, which is taken from the 3D numerical simulation (as explained below). For a monoatomic perfect gas, one has $q=3 / 2$, hence $r=-1 / 6$. However, our 3D numerical simulations indicate a value of $r$ closer to 0 . The reason is likely that there is radiative cooling. Hence, $\gamma\rangle \Gamma=c_{P} / c_{V}$, where $\gamma$ is the polytropic index $(q=1 /(\gamma-1))$.

Following Rieutord \& Zahn (1995), we assume that all the convective energy flux is transported by the plume, thus

$F=L_{\odot} / N$,

where $N$ is the number of plumes in the shell at $h=R_{\odot}-z$. We find $N \approx 6 \times 10^{6}$ from the $3 \mathrm{D}$ numerical simulation. To obtain such a result, one has to use the relation between $a$ and $N$ :

$a=N \pi b_{\mathrm{p}}^{2} / 4 \pi h^{2}$,

where $(a)$ is mean fractional area of the upflow, $h=R_{\odot}-z$, and $b_{\mathrm{p}}$ is the radius of a plume. $\left(b_{\mathrm{p}}\right)$ and $a$ are taken from the $3 \mathrm{D}$ numerical simulation. We assume $a=0.7$, as taken from Fig. 1, which shows that the mean fractional area $a$ is roughly constant in the quasi-adiabatic convection zone. The plume radius, $b_{\mathrm{p}}$, is estimated at the top of the simulated box, which corresponds to the photosphere.

At this stage, we have modeled the downdrafts, but not yet the updrafts. The 3D numerical simulations show that mean velocities of upflow and downflow obey the same power law (Fig. 6). This can be explained as follows: from the conservation of the mass flux one has

$\langle\rho w\rangle=a\langle\rho w\rangle_{\mathrm{u}}+(1-a)\langle\rho w\rangle_{\mathrm{d}}=0$.

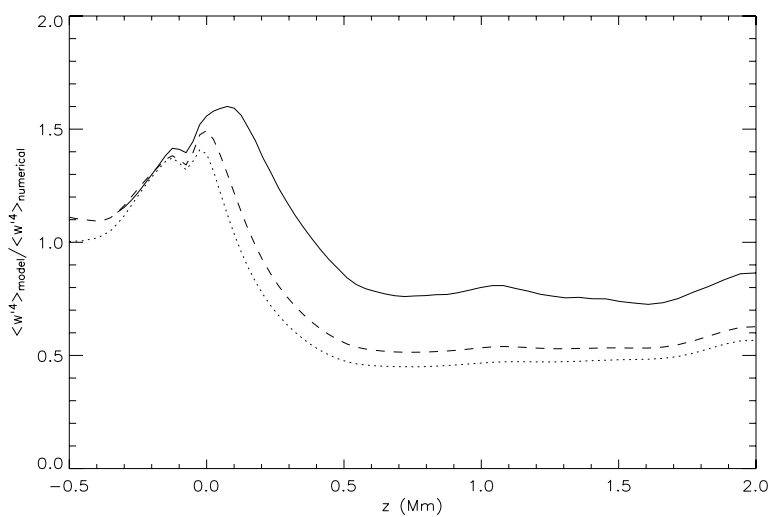

Fig. 7. Fourth-order moment $\left\langle w^{\prime 4}\right\rangle$ as a function of depth $z$ normalized to the FOM calculated directly from numerical simulations. The solid line shows $\left\langle w^{\prime 4}\right\rangle$ calculated using the CMP model, the dashed line is the moment as obtained from Eq. (14) with Eq. (10) for $S_{w}$, and the dotted line is the QNA, Eq. (26).

Fluctuating parts of densities in up and downflows are neglected such that $\rho_{\mathrm{u}} \approx\langle\rho\rangle_{\mathrm{u}}$ and $\rho_{\mathrm{d}} \approx\langle\rho\rangle_{\mathrm{d}}$ (see Fig. 2b of RZ95). Thus,

$\langle w\rangle_{\mathrm{u}}=-\frac{(1-a)}{a} \frac{\langle\rho\rangle_{\mathrm{d}}}{\langle\rho\rangle_{\mathrm{u}}}\langle w\rangle_{\mathrm{d}}$.

Then, assuming that $\langle\rho\rangle_{\mathrm{u}}=\rho_{\mathrm{u} 0}\left(z / z_{0}\right)^{q}$ and $\langle\rho\rangle_{\mathrm{d}}=\rho_{\mathrm{d} 0}\left(z / z_{0}\right)^{q}$ obey the same power law as in Eq. (36):

$\langle w\rangle_{\mathrm{u}} \approx-\frac{(1-a)}{a} \frac{\rho_{\mathrm{d} 0}}{\rho_{u 0}}\langle w\rangle_{\mathrm{d}}$

$\rho_{\mathrm{u} 0}$ and $\rho_{\mathrm{d} 0}$ are the values at the reference depth $z_{0}$. We set $a \approx 0.7$ (see Fig. 1), which is the value obtained in the quasiadiabatic zone from the 3D numerical simulation. Assuming further that $\rho_{\mathrm{d} 0} / \rho_{\mathrm{u} 0} \approx 1$, one obtains

$\left|\langle w\rangle_{\mathrm{u}}\right| \approx 0.45\left|\langle w\rangle_{\mathrm{d}}\right|$

which is approximately what is seen in Fig. 6.

\subsubsection{Skewness $S_{w}$ and the fourth-order moment}

We use Eq. (33) for the skewness with

$\delta w=\left(\left\langle w_{\mathrm{u}}\right\rangle-\left\langle w_{\mathrm{d}}\right\rangle\right) \approx 1.45 w_{\mathrm{d} 0}$.

The vertical depth of the computation box is narrow in comparison with the reference depth $z_{0}$, thus $\delta w$ varies only weakly with $z$. Hence, we assume $r=0$ in the solar case. The fourthorder moment $\left\langle w^{\prime 4}\right\rangle$ can then be computed by means of the interpolated relation Eq. (14). In Fig. 7, we show the resulting $\left\langle w^{\prime 4}\right\rangle$. The CMP clearly is an improvement compared to the QNA and the TFM expression for $S_{w}$, Eq. (10) combined with Eq. (14), by at least a factor two in the quasi-adiabatic zone. The FOM in the superadiabatic zone is overestimated. Indeed, as mentioned above, the CMP is not able to describe such a zone mainly because the assumptions of Sect. 4.1 are not valid. Note that it is possible to use the same procedure to compute any other thirdand fourth-order moment.

\subsubsection{Determination of $\delta \theta$}

Similarly to the procedure in the previous section, we evaluate $\left\langle\tilde{\theta}^{\prime 2}\right\rangle$ with the help of Eqs. (32) and (34). We therefore need to 


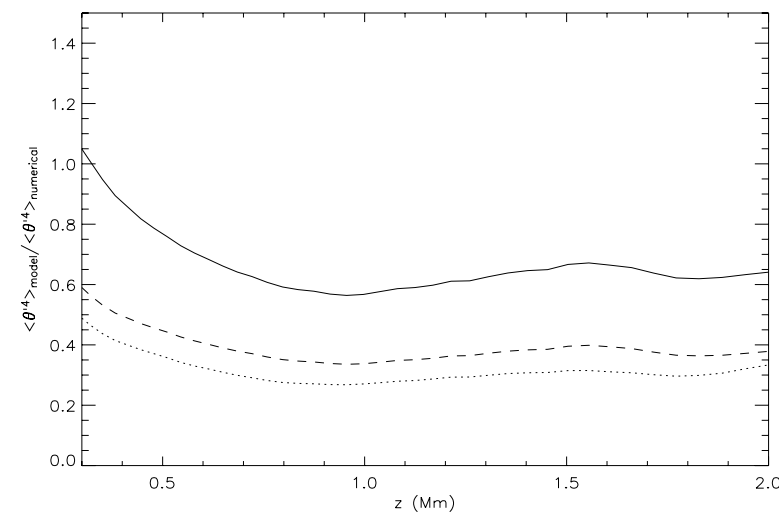

Fig. 8. Fourth-order moment $\left\langle\theta^{\prime 4}\right\rangle$ as a function of depth $z$ normalized to the directly numerically calculated FOM. In solid lines the moment stems from $\left\langle\theta^{\prime 4}\right\rangle$ calculated using the CMP model, the dashed line is the moment as obtained from Eqs. (14) and (10) for $S_{\theta}$ and the dotted line is the QNA.

determine $\delta \theta$. The temperature profile is more sensitive to departure from adiabaticity than the velocity profile. It is therefore not suitable to assume an isentropic envelope. Such an approximation can still be used in the downflow, but not for the upflow, which is far from being adiabatic due to radiative cooling. Then, for the sake of simplicity, we assume a power law to obtain $\delta \theta$ :

$\delta \theta \approx \delta \theta_{0}\left(\frac{z}{z_{0}}\right)^{m}$

For $z>1 \mathrm{Mm}$ in the simulated box $(z=0 \mathrm{Mm}$ denotes the photosphere), one derives $m=-1.5, \delta \theta_{0} \approx 170 \mathrm{~K}$ from the 3D numerical simulation. Using the power law (Eq. (46)) with $m=-1.5$, the skewness $S_{\theta}$ can be calculated using Eq. (34). In Fig. 8, we present the fourth-order moment $\left\langle\theta^{\prime 4}\right\rangle$ computed using the CMP, and as expected, the description of the FOM is improved. In the deeper part of the convection zone (i.e., the adiabatic region), $\delta \theta$ is easier to model because Eq. (36) can be used and the difference $\delta \theta$ becomes a power law. From Eqs. (8), (14), (33), and (34) all the third- and fourth-order moments can be modelled with the CMP.

\subsubsection{Summary: the CMP in a nutshell}

In practice, one uses the CMP to compute $\left\langle w^{\prime 4}\right\rangle$ by means of the interpolation formula Eq. (14), where the second-order moment $\left\langle w^{\prime 2}\right\rangle$ is supposed to be known and where the skewness $S_{w}$ is computed from Eq. (33). In the latter expression, $\delta w$ is determined using the plume model through Eq. (45) and using Eqs. (37) to (40) with appropriate values of parameters for the case studied (in the present paper we used the values from Table 1, which are suitable for the solar case). Here, $a(z), N$, $b_{\mathrm{p}}$, and other input quantities are taken from the 3D numerical simulation. When the CMP is used to obtain the other thirdand fourth-order moments, additional quantities have to be determined, namely $b$ and $m$ in Eq. (46) for $S_{\theta}$ (see Eq. (34)).

\section{Conclusions}

With the help of 3D numerical simulations of the upper part of the solar convective region, we have shown that the QNA and the TFM fail to describe the fourth-order velocity and temperature correlation moments, if merely used on their own. These results confirm KR2006 and geophysical studies (Gryanik \& Hartmann 2002) and led us to generalize the TFM in order to take the effects of the turbulent properties of the up- and downflows explicitly into account (GTFM). We point out that the GTFM can be used in other contexts than the solar one as long as the convective system can be described with two turbulent flows.

One might wonder whether it is likely that the CMP and the model for $p$ mode excitation developed in Paper II are generally applicable to solar-like stars. To answer this question requires further work, but results on important ingredients of these models are encouraging. The case of convection in the planetary boundary layer of the atmosphere of the earth was already discussed in GH2002. Their interpolation model for FOMs has meanwhile been investigated for the case of convection in the ocean (Losch 2004) and solar granulation (Kupka \& Robinson 2006, who also study the case of a K dwarf; preliminary results were published in Kupka \& Hillebrandt 2005). We corroborate the latter here with simulations for solar granulation based on more realistic boundary conditions. The overall conclusion that can be drawn from these studies is that, at least away from the boundary layers of convection zones, the FOMs in purely convective flows can be estimated according to the interpolation model by GH2002 with an accuracy typically in the range of $20 \%$ to $30 \%$, whereas the QNA is off by a factor of two to three. For the superadiabatic layer, the discrepancies of the QNA remain the same in any case of the same size.

We focused here on the solar case, more precisely a region that is nearly adiabatic, just below the superadiabatic zone where the acoustic modes are excited. As indicated by the 3D simulations, the coherent downdrafts, called plumes, are more turbulent than the upflow. In addition, we use the plume model developed by RZ95 to estimate the upward and downward mean velocities. With these additional approximations, the GTFM yields a closure model, the CMP, which can be applied in the quasi-adiabatic zone (located just below the superadiabatic one). Comparisons of calculations based on the CMP with direct calculations from the 3D numerical simulations show a good agreement. Hence, the CMP provides an analytical closure for third- and fourthorder moments. These moments are expressed in a simple way and require only the knowledge of the second-order moments and the parameters of the plume model. We stress that the CMP involves four parameters: the number of plumes in the considered shell (i.e., near the photosphere), the exponent of the power law for the mean vertical velocity of plumes, the law to describe the temperature difference between the two flows, and the mean fractional area of the updrafts and hot drafts.

A study of the dependence of the results on these parameters is in progress. For instance, an increase of $a$ will imply an increase of $S_{w}$ in Eq. (33), and hence of the fourth-order moment $\left\langle w^{\prime 4}\right\rangle$. Nevertheless, it is extremely difficult to deduce the behaviour of the system, since from Eq. (41) a variation of $a$ changes the velocities of the flows. Instead, one could use a set of numerical simulations to study the effect of a change of the parameter $a$. In a companion paper, we use the CMP in a semianalytical approach to calculate the power supplied to the solar $p$ modes. It is found that the power is quite significantly affected by the adopted closure model.

Our final aim is to apply the CMP to the study of stochastic excitation of solar-like $p$ modes in stars other than the Sun. It will be necessary to assess the validity of the CMP approximations to extend their application to stellar conditions different from the solar case. This will also require investigating the dependence of the parameters entering the CMP, for instance, on the effective temperature of the star (work which is in progress). As pointed 
out in Sect. 4, the CMP is valid only in the quasi-adiabatic zone due to the power laws used to model the plume dynamics. This will be discussed further in the companion paper in which the present model will be used in the superadiabatic zone in order to propose a new closure for the calculation of stellar $p$ modes.

Finally, we note that in the present work we do not take the effect of differential rotation and meridional circulation into account. However, recent helioseismic investigations (Schou et al. 2002; Zhao \& Kosovichev 2004) have shown that variability of those large-scale flows gradually affects wavelength and frequencies, leading to a redistribution of the observed power spectrum (Shergelashvili \& Poedts 2005; Hindman et al. 2005). Hence, it could have an indirect effect on the amplitudes of $p$ modes. Furthermore, large-scale laminar non-uniform flows can have a significant effect on the formation of the coherent structures and intrinsic turbulence (Miesch et al. 2000; Brun \& Toomre 2002; Rempel 2005). To what extent they can affect solar $p$ mode amplitudes, through the closure model and the Reynolds stresses, remains to be investigated.

Acknowledgements. We are indebted to J. Leibacher for his careful reading of the manuscript and his helpful remarks. We thank J. P. Zahn and F. Lignières for useful discussions and comments. F.K. is grateful to V. M. Gryanik and J. Hartmann for discussions on their model and their observational data. We also thank the anonymous referee for valuable comments that helped to improve the manuscript.

We thank Å. Nordlund and R. F. Stein for making their code available to us. Their code was made at the National Center for Supercomputer Applications and Michigan State University and supported by grants from NASA and NSF.

\section{References}

Abdella, K., \& McFarlane, N. 1997, J. Atm. Sci., 54, 1850

Balmforth, N. J. 1992, MNRAS, 255, 639
Belkacem, K., Samadi, R., Goupil, M., Kupka, F., \& Baudin, F. 2006, A\&A, 460, 183

Brun, A. S., \& Toomre, J. 2002, ApJ, 570, 865

Canuto, V. M. 1992, ApJ, 392, 218

Canuto, V. M., \& Dubovikov, M. 1998, ApJ, 493, 834

Cattaneo, F., Brummell, N. H., Toomre, J., Malagoli, A., \& Hurlburt, N. E. 1991, ApJ, 370, 282

Goldreich, P., \& Keeley, D. A. 1977, ApJ, 212, 243

Goldreich, P., Murray, N., \& Kumar, P. 1994, ApJ, 424, 466

Gryanik, V. M., \& Hartmann, J. 2002, J. Atm. Sci., 59, 2729

Gryanik, V. M., Hartmann, J., Raasch, S., \& Schröter, M. 2005, J. Atm. Sci., 62, 2632

Hindman, B. W., Gough, D., Thompson, M. J., \& Toomre, J. 2005, ApJ, 621, 512

Kupka, F., \& Hillebrandt, W. 2005, Workshop on Interdisciplinary Aspects of Turbulence

Kupka, F., \& Robinson, F. 2006, MNRAS, in press

Lesieur, M. 1997, Turbulence in fluids (Kluwer Academic Publishers)

Losch, M. 2004, Geophys. Res. Lett., 31, 23301

Miesch, M. S., Elliott, J. R., Toomre, J., et al. 2000, ApJ, 532, 593

Millionshchikov, M. D. 1941, Doklady Acad. Nauk SSSR, 32, 611

Nordlund, Å., \& Stein, R. F. 2001, ApJ, 546, 576

Ogura, Y. 1963, J. Fluid Mech., 16, 33

Petersen, A. C., Beets, C., van Dop, H., Duynkerke, P. G., \& Pier Siebesma, A. 1999, J. Atm. Sci., 56, 37

Randall, D. A., Shao, Q., \& Moeng, C.-H. 1992, J. Atm. Sci., 49, 1903

Rempel, M. 2005, ApJ, 631, 1286

Rieutord, M., \& Zahn, J.-P. 1995, A\&A, 296, 127

Samadi, R., \& Goupil, M. . 2001, A\&A, 370, 136

Samadi, R., Goupil, M.-J., Alecian, E., et al. 2005, J. Astrophys. Atr., 26, 171

Schou, J., Howe, R., Basu, S., et al. 2002, ApJ, 567, 1234

Shergelashvili, B. M., \& Poedts, S. 2005, A\&A, 438, 1083

Siebesma, A. P., \& Cuijpers, J. W. M. 1995, J. Atmos. Sci., 52, 650

Stein, R., Georgobiani, D., Trampedach, R., Ludwig, H.-G., \& Nordlund, Å. 2004, Sol. Phys., 220, 229

Stein, R. F., \& Nordlund, A. 1998, ApJ, 499, 914

Turner, J. S. 1986, J. Fluid Mech., 173, 431

Zhao, J., \& Kosovichev, A. G. 2004, ApJ, 603, 776 
K. Belkacem et al.: A closure model with plumes. I., Online Material p 1

\section{Online Material}




\section{Appendix A: Cross-correlation moments}

As explained in Sect. 3.1, we provide the cross-correlation moments:

$$
\begin{aligned}
\left\langle w^{\prime} \theta^{\prime}\right\rangle= & a_{\mathrm{uh}}\left\langle\tilde{w}^{\prime} \tilde{\theta}^{\prime}\right\rangle_{\mathrm{u}, \mathrm{h}}+a_{\mathrm{uc}}\left\langle\tilde{w}^{\prime} \tilde{\theta}^{\prime}\right\rangle_{\mathrm{u}, \mathrm{c}} \\
& +a_{\mathrm{dc}}\left\langle\tilde{w}^{\prime} \tilde{\theta}^{\prime}\right\rangle_{\mathrm{d}, \mathrm{c}}+a_{\mathrm{dh}}\left\langle\tilde{w}^{\prime} \tilde{\theta}^{\prime}\right\rangle_{\mathrm{d}, \mathrm{h}}+\eta \delta w \delta \theta \\
\left\langle w^{\prime 2} \theta^{\prime}\right\rangle= & a_{\mathrm{uh}}\left\langle\tilde{w}^{\prime 2} \tilde{\theta}^{\prime}\right\rangle_{\mathrm{u}, \mathrm{h}}+a_{\mathrm{uc}}\left\langle\tilde{w}^{\prime 2} \tilde{\theta}^{\prime}\right\rangle_{\mathrm{u}, \mathrm{c}} \\
& +a_{\mathrm{dh}}\left\langle\tilde{w}^{\prime 2} \tilde{\theta}^{\prime}\right\rangle_{\mathrm{d}, \mathrm{h}}+a_{\mathrm{dc}}\left\langle\tilde{w}^{\prime 2} \tilde{\theta}^{\prime}\right\rangle_{\mathrm{d}, \mathrm{c}} \\
& +\beta_{1} \delta \theta+\beta_{2} \delta w+\beta_{3} \delta w^{2} \delta \theta \\
\left\langle w^{\prime} \theta^{\prime 2}\right\rangle= & a_{\mathrm{uh}}\left\langle\tilde{w}^{\prime} \tilde{\theta}^{\prime 2}\right\rangle_{\mathrm{u}, \mathrm{h}}+a_{\mathrm{uc}}\left\langle\tilde{w}^{\prime} \tilde{\theta}^{\prime 2}\right\rangle_{\mathrm{u}, \mathrm{c}} \\
& +a_{\mathrm{dh}}\left\langle\tilde{w}^{\prime} \tilde{\theta}^{\prime 2}\right\rangle_{\mathrm{d}, \mathrm{h}}+a_{\mathrm{dc}}\left\langle\tilde{w}^{\prime} \tilde{\theta}^{\prime 2}\right\rangle_{\mathrm{d}, \mathrm{c}} \\
& +\gamma_{1} \delta w+\gamma_{2} \delta \theta+\gamma_{3} \delta w \delta \theta^{2} \\
\left\langle w^{\prime 2} \theta^{\prime 2}\right\rangle= & a_{\mathrm{uh}}\left\langle\tilde{w}^{\prime 2} \tilde{\theta}^{\prime 2}\right\rangle_{\mathrm{u}, \mathrm{h}}+a_{\mathrm{uc}}\left\langle\tilde{w}^{\prime 2} \tilde{\theta}^{\prime 2}\right\rangle_{\mathrm{u}, \mathrm{c}} \\
& +a_{\mathrm{dh}}\left\langle\tilde{w}^{\prime 2} \tilde{\theta}^{\prime 2}\right\rangle_{\mathrm{d}, \mathrm{h}}+a_{\mathrm{dc}}\left\langle\tilde{w}^{\prime 2} \tilde{\theta}^{\prime 2}\right\rangle_{\mathrm{d}, \mathrm{c}} \\
& +\phi_{1} \delta \theta+\phi_{2} \delta w+\phi_{3} \delta \theta^{2}+\phi_{4} \delta w^{2} \\
& +\phi_{5} \delta w \delta \theta+\phi_{6} \delta w^{2} \delta \theta^{2}
\end{aligned}
$$

where:

$$
\begin{aligned}
\eta= & {\left[a_{\mathrm{uh}}(1-a)(1-b)-a_{\mathrm{uc}} b(1-a)+a_{\mathrm{dc}} a b-a_{\mathrm{dh}} a(1-b)\right] } \\
\beta_{1}= & {\left[a_{\mathrm{uh}}(1-b)-a_{\mathrm{uc}} b\right]\left\langle\tilde{w}^{\prime 2}\right\rangle_{\mathrm{u}} } \\
& +\left[a_{\mathrm{dh}}(1-b)-a_{\mathrm{dc}} b\right]\left\langle\tilde{w}^{\prime 2}\right\rangle_{\mathrm{d}} \\
\beta_{2}=2 & {\left[a_{\mathrm{uh}}\left\langle\tilde{w}^{\prime} \tilde{\theta}^{\prime}\right\rangle_{\mathrm{u}, \mathrm{h}}+a_{\mathrm{uc}}\left\langle\tilde{w}^{\prime} \tilde{\theta}^{\prime}\right\rangle_{\mathrm{u}, \mathrm{c}}-a\left(\left\langle w^{\prime} \theta^{\prime}\right\rangle-\eta\right)\right] } \\
\beta_{3}= & {\left[a_{\mathrm{uh}}(1-a)^{2}(1-b)-a_{\mathrm{uc}} b(1-a)^{2}+a_{\mathrm{dh}} a^{2}(1-b)-a_{\mathrm{dc}} a^{2} b\right] }
\end{aligned}
$$

$$
\gamma_{1}=\left[a_{\mathrm{uh}}(1-a)-a_{\mathrm{dh}} a\right]\left\langle\tilde{\theta}^{\prime 2}\right\rangle_{\mathrm{h}}
$$$$
+\left[a_{\mathrm{uc}}(1-a)-a_{\mathrm{dc}} a\right]\left\langle\tilde{\theta}^{\prime 2}\right\rangle_{\mathrm{c}}
$$$$
\gamma_{2}=2\left[a_{\mathrm{uh}}\left\langle\tilde{w}^{\prime} \tilde{\theta}^{\prime}\right\rangle_{\mathrm{u}, \mathrm{h}}+a_{\mathrm{dh}}\left\langle\tilde{w}^{\prime} \tilde{\theta}^{\prime}\right\rangle_{\mathrm{d}, \mathrm{h}}-b\left(\left\langle w^{\prime} \theta^{\prime}\right\rangle-\eta\right)\right]
$$$$
\gamma_{3}=\left[a_{\mathrm{uh}}(1-a)(1-b)^{2}-a_{\mathrm{uc}} b^{2}(1-a)-a_{\mathrm{dh}} a(1-b)^{2}-a_{\mathrm{dc}} a b^{2}\right]
$$$$
\phi_{1}=2\left[a_{\mathrm{uh}}\left\langle\tilde{w}^{\prime 2} \tilde{\theta}^{\prime}\right\rangle_{\mathrm{u}, \mathrm{h}}+a_{\mathrm{dh}}\left\langle\tilde{w}^{\prime 2} \tilde{\theta}^{\prime}\right\rangle_{\mathrm{d}, \mathrm{h}}\right.
$$$$
\left.-b\left(\left\langle w^{\prime 2} \theta^{\prime}\right\rangle-\beta_{1}-\beta_{2}-\beta_{3}\right)\right]
$$$$
\phi_{2}=2\left[a_{\mathrm{uh}}\left\langle\tilde{w}^{\prime} \tilde{\theta}^{\prime 2}\right\rangle_{\mathrm{u}, \mathrm{h}}+a_{\mathrm{uc}}\left\langle\tilde{w}^{\prime 2} \tilde{\theta}^{\prime}\right\rangle_{\mathrm{u}, \mathrm{c}}\right.
$$$$
\left.-a\left(\left\langle w^{\prime} \theta^{\prime 2}\right\rangle-\gamma_{1}-\gamma_{2}-\gamma_{3}\right)\right]
$$$$
\phi_{3}=\left[a_{\mathrm{uh}}(1-b)^{2}-a_{\mathrm{uc}} b^{2}\right]\left\langle\tilde{w}^{2}\right\rangle_{\mathrm{u}}
$$$$
+\left[a_{\mathrm{dh}}(1-b)^{2}-a_{\mathrm{dc}} b^{2}\right]\left\langle\tilde{w}^{\prime 2}\right\rangle_{\mathrm{d}}
$$

$\phi_{4}=\left[a_{\mathrm{uh}}(1-a)^{2}-a_{\mathrm{dh}} a^{2}\right]\left\langle\tilde{\theta}^{\prime 2}\right\rangle_{\mathrm{h}}$

$$
+\left[a_{\mathrm{uc}}(1-a)^{2}-a_{\mathrm{dc}} a^{2}\right]\left\langle\tilde{\theta}^{\prime 2}\right\rangle_{\mathrm{c}}
$$$$
\phi_{5}=4\left[a_{\mathrm{uh}}(1-a)(1-b)\left\langle\tilde{w}^{\prime} \tilde{\theta}^{\prime}\right\rangle_{\mathrm{u}, \mathrm{h}}-a_{\mathrm{uc}} b(1-a)\left\langle\tilde{w}^{\prime} \tilde{\theta}^{\prime}\right\rangle_{\mathrm{u}, \mathrm{c}}\right.
$$$$
\left.-a_{\mathrm{dh}} a(1-b)\left\langle\tilde{w}^{\prime} \tilde{\theta}^{\prime}\right\rangle_{\mathrm{d}, \mathrm{h}}+a_{\mathrm{dc}} a b\left\langle\tilde{w}^{\prime} \tilde{\theta}^{\prime}\right\rangle_{\mathrm{d}, \mathrm{c}}\right]
$$

$\phi_{6}=\left[a_{\mathrm{uh}}(1-a)^{2}(1-b)^{2}+a_{\mathrm{uc}}(1-a)^{2} b^{2}+a_{\mathrm{dh}} a^{2}(1-b)^{2}\right.$

$$
\left.+a_{\mathrm{dc}} a^{2} b^{2}\right]
$$

